\title{
Improved Surgical Technique for Laparoscopic Roux-en-Y Gastric Bypass Reduces Complications at the Gastrojejunostomy
}

\author{
Michel Suter • Andrea Donadini • Jean-Marie Calmes • \\ Sébastien Romy
}

Published online: 5 May 2010

(C) Springer Science+Business Media, LLC 2010

\begin{abstract}
Roux-en-Y gastric bypass (RYGBP) is one of the most commonly performed bariatric procedures for morbidly obese patients. It is associated with effective longterm weight loss, but can lead to significant complications, especially at the gastrojejunostomy (GJS). All the patients undergoing laparoscopic RYGBP at one of our two institutions were included in this study. The prospectively collected data were reviewed retrospectively for the purpose of this study, in which we compared two different techniques for the construction of the GJS and their effects on the incidence of complications. In group A, anastomosis was performed on the posterior aspect of the gastric pouch. In group $\mathrm{B}$, it was performed across the staple line used to form the gastric pouch. A 21-mm circular stapler was used in all patients. A total of 1,128 patients were included between June 1999 and September 2009-639 in group A and 488 in group B. Sixty patients developed a total of 65 complications at the GJS, with 14 (1.2\%) leaks, $42(3.7 \%)$ strictures, and $9(0.8 \%)$ marginal ulcers. Leaks $(0.2 \%$ versus $2 \%, p=0.005)$ and strictures $(0.8 \%$ versus $5.9 \%$, $p<0.0001)$ were significantly fewer in group B than in group A. Improved surgical technique, as we propose, with the GJS across the staple line used to form the gastric pouch, significantly reduces the rate of anastomotic complications at the GJS. A circular 21-mm stapler can be used with a low complication rate, and especially a low
\end{abstract}

\footnotetext{
M. Suter

Department of Surgery, Hôpital du Chablais,

1860 Aigle-Monthey, Switzerland

M. Suter $(\varangle) \cdot$ A. Donadini $\cdot$ J.-M. Calmes $\cdot$ S. Romy

Department of Visceral Surgery,

Centre Hospitalier Universitaire Vaudois,

Lausanne, Switzerland

e-mail: michelsuter@netplus.ch
}

stricture rate. Additional methods to limit complications at the GJS are probably not routinely warranted.

Keywords Obesity surgery - Gastric bypass .

Surgical technique $\cdot$ Complications

\section{Introduction}

The prevalence of morbid obesity is increasing worldwide, resulting in an ever-increasing number of patients being submitted to bariatric surgery, and especially Roux-en-Y gastric bypass (RYGBP), which is considered as the gold standard procedure in this setting. Since its introduction in 1994, the safety of laparoscopic RYGBP has been shown to be at least equal, if not superior, to that of open RYGBP. Laparoscopic RYGBP, however, caries a given morbidity, and complications purely related to the procedure (leaks, hemorrhage, stricture, and occlusion) still represent a large percentage of the total complication rate and are responsible for prolonged hospital stay, increased cost, and mortality.

Early- and long-term complications at the gastrojejunostomy are not uncommon after laparoscopic RYGBP, with leaks occurring in up to $8 \%$ and strictures in up to $30 \%$ of the patients [1-23]. Their incidence is, at least in part, related to the precise surgical technique used to perform the anastomosis and the so-called learning curve. Among technical aspects in the creation of the gastrojejunostomy are the type of anastomosis (hand-sutured versus stapled), the type of staples (circular versus linear), the route chosen for the alimentary limb, which can result in more or less tension (ante- versus retro-colic), the type of sutures used either to perform the suture or to reinforce the anastomosis (absorbable versus nonabsorbable). Various recommendations to decrease the incidence of anastomotic complications have been made, and 


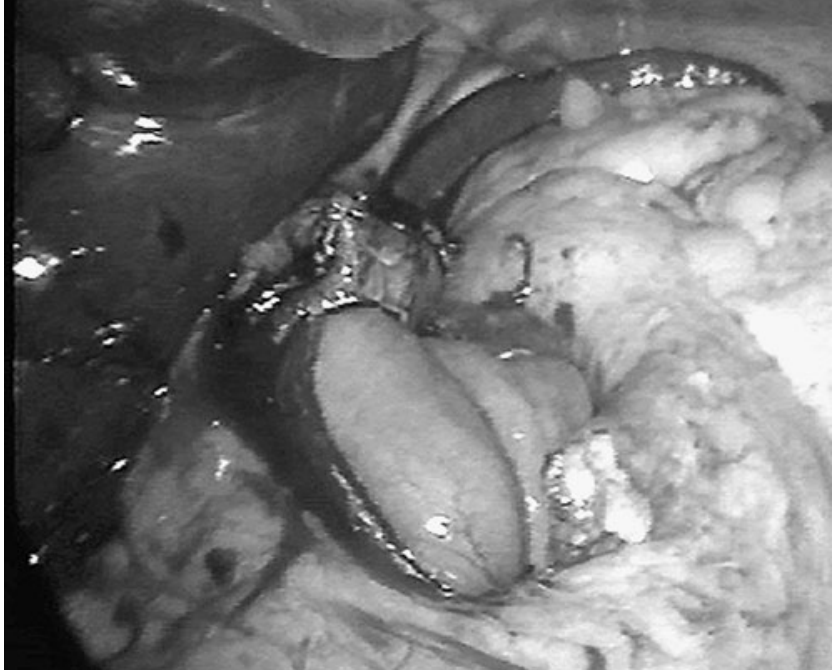

Fig. 1 Gastrojejunostomy in group A: circular anastomosis posterior to the pouch staple line with ischemic tissue band between the two staple lines

include using larger circular staplers, reinforcing the suture lines or using fibrin sealant, among others.

In this paper, we present our results with two circular stapling techniques for the gastrojejunostomy in an otherwise very homogenous large series of RYGBP patients operated between 1999 and 2009 and show that improved surgical technique in the more recent years resulted in a significant decrease in the overall incidence of anastomotic complications at this level.

\section{Patients and Methods}

Patients with a body mass index (BMI) in excess of $40 \mathrm{~kg} / \mathrm{m}^{2}$ or $>35 \mathrm{~kg} / \mathrm{m}^{2}$ with at least one severe comorbidity, were offered laparoscopic Roux-en-Y gastric bypass after failure of conservative treatment, complete standardized evaluation by a multidisciplinary team, and education during a threesession course on bariatric surgery [24]. Contraindications were according to the consensus development conference panel of the National Institute of Health and to the consensus on obesity treatment in Switzerland $[25,26]$.

At the induction of anaesthesia, the patients were given prophylactic antibiotics (2.2 g amoxicillin-clavulanate or $200 \mathrm{mg}$ ciprofloxacine) intravenously. Prophylaxis against thromboembolism was started either the evening before surgery, or at the induction of anaesthesia using low molecular weight heparin at a dose that was adapted to the patient's body weight and pursued until the end of the second post-operative week. Proton pump inhibitors were started the day after surgery and maintained for 1 month in order to prevent the development of marginal ulceration.
All operations were performed using six trocars and a $45^{\circ}$-angled optic, with the patient in a steep reverse Trendelenburg position, and the surgeon between the patient's legs. The stomach was divided just below the cardia using a linear stapler with blue cartridges in order to form a proximal gastric pouch of approximately $10-15 \mathrm{ml}$. The jejunum was divided between 20 and $40 \mathrm{~cm}$ from the angle of Treitz using the linear stapler with a white cartridge. The Roux limb was brought close to the gastric pouch in a retrocolic and retrogastric fashion. The anastomosis between the gastric pouch and the Roux limb was performed using a $21-\mathrm{mm}$ circular stapler, the anvil of which was introduced in the gastric pouch transorally. The jejunojejunostomy was done side-to-side using a linear stapler with a white cartridge, and the proximal end of the Roux limb was staple-closed using the same instrument. The length of the Roux-en-Y limb was determined according to the patient's BMI-100 $\mathrm{cm}$ up to a BMI of 48 and $150 \mathrm{~cm}$ with a BMI $>48 \mathrm{~kg} / \mathrm{m}^{2}$. All the mesenteric windows were closed, and a Jackson-Pratt drain was left close to the gastrojejunostomy at the end of the procedure.

During our experience, the gastrojejunostomy was always performed with a 21-mm circular stapler, but the precise technique was modified over time; in our initial experience, encompassing 639 patients (group A), the anastomosis was placed on the posterior wall of the gastric pouch (Fig. 1), and the entire circular staple line remained behind the transverse staple line used to divide the pouch. This usually left a small portion of gastric wall between the two staple lines. More recently, in the last 488 patients (group B), the anastomosis was placed across the staple line used to divide the gastric pouch, usually at the angle between the first and second firings, thereby avoiding to leave a narrow band of ischemic tissue between the two staple lines (Fig. 2). A few additional absorbable sutures,

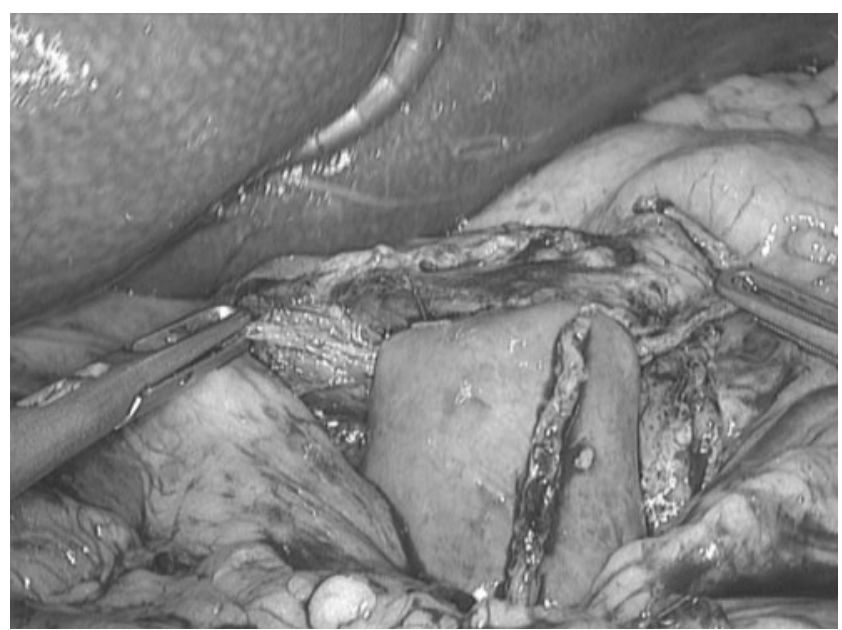

Fig. 2 New form of gastrojejunostomy in group B: circular anastomosis across the pouch staple line 
Table 1 Preoperative characteristics of the two patient groups

\begin{tabular}{lccc}
\hline & Group A & Group B & $p$ value \\
\hline Number & 639 & 488 & \\
Male/female (\%) & $143(22.4 \%)$ & $120(24.6 \%)$ & 0.39 \\
Reoperative procedures (\%) & $61(9.5 \%)$ & $51(10.4 \%)$ & 0.61 \\
Mean age (extremes) & $39.8(18-64)$ & $41.1(18-66)$ & 0.036 \\
Mean BMI (extremes) & $44.9(17.7-73.4)$ & $45.2(27.6-76.6)$ & 0.52 \\
Conversions & 5 & 2 & 0.7 \\
\hline
\end{tabular}

usually three, were placed in all the patients between the jejunal loop and the gastric pouch to release any possible tension on the anastomosis.

The patients underwent a water-soluble contrast study on post-operative day 1 not only in search for a possible leak but also to ensure that passage through the anastomosis was adequate. If a leak was detected, treatment was according to the size of the leak and the patient's condition. If the study proved negative, the patients were slowly advanced on a semi-liquid diet, which was maintained for a month. After that, patients were instructed to progressively return to normal food.

Patients who complained from unusual dysphagia during follow-up were submitted to repeat contrast study and/or endoscopy to ascertain the diagnosis of stenosis and to exclude marginal ulceration. Those with severe dysphagia preventing proper hydration were offered endoscopic dilation, whereas those with only moderate dysphagia were treated conservatively and remained on a soft diet an additional few weeks. Only stenoses requiring dilatation were considered for analysis in this study.

All bariatric patients' data have been entered prospectively in a computerized database since 1995. These data were analysed retrospectively for the purpose of this study. Statistical analysis was performed using the $t$ test for continuous variables and the $\chi^{2}$ test of the Fisher's exact test for categorical variables, as appropriate. Statistical significance was accepted with a $p$ level $\leq 0.05$.

\section{Results}

Between June 1999 and September 2009, 1,128 patients underwent laparoscopic Roux-en-Y gastric bypass in our two institutions. Of these, 1,016 were primary operations and 112 were reoperations after failed and/or complicated prior bariatric procedures. There were 263 men and 865 women, with a mean age of 40.3 years (18-66). The mean
BMI was $45.1 \mathrm{~kg} / \mathrm{m}^{2}$ (17.7-76.2). Conversion was necessary in seven patients, of whom five were excluded from further analysis because their gastrojejunostomy was hand-sutured in an open fashion. Table 1 shows the preoperative characteristics of our patients. Patients in group B were slightly older those in group A (41.1 versus 39.8 years, $p=$ 0.036 ), but the groups were otherwise comparable regarding the male/female ratio, proportion of reoperations, and BMI.

A total of 65 complications developed at the gastrojejunostomy in 60 patients. There were a total of $14(1.2 \%)$ leaks and $42(3.7 \%)$ anastomotic strictures. Three patients sequentially developed both complications. Nine $(0.8 \%)$ patients developed marginal ulceration. Of these, one occurred 3 weeks postoperatively, with significant haemorrhage requiring transfusion of two blood units, whereas eight developed in the long term. In group A, leaks and stenosis were evenly distributed over time and did not significantly decrease with experience (Table 2). Overall, there were more anastomotic complications in group A than in group B. Table 3 show the distribution of complications in the two patient groups for primary and reoperative procedures. Patients undergoing primary RYGBP in group B developed significantly fewer leaks $(0.2 \%$ versus $1.7 \%, p=$ $0.02)$ and fewer strictures $(0.7 \%$ versus $5.7 \%, p<0.0001)$ than patients in group A. The number of anastomotic complications was also higher in reoperative procedures, but the differences did not reach statistical significance.

\section{Discussion}

Early- and long-term complications at the gastrojejunostomy are not uncommon after laparoscopic RYGBP. They include leaks, strictures, and marginal ulcers. Leaks are typically an early complication. They develop within the first few days after surgery and can sometimes be devastating if not treated promptly and aggressively, although they may be completely asymptomatic. Marginal
Table 2 Distribution of leaks in group A according to experience

\begin{tabular}{llllllll}
\hline Patient \# & $1 / 100$ & $101 / 200$ & $201 / 300$ & $301 / 400$ & $401 / 500$ & $501 / 600$ & $>600$ \\
\hline Number of leaks & 2 & 3 & 1 & 2 & 2 & 2 & 1 \\
$\%$ & 2 & 3 & 1 & 2 & 2 & 2 & 2.5 \\
\hline
\end{tabular}


Table 3 Gastrojejunostomy complications in primary and reoperative procedures

\begin{tabular}{lccc}
\hline & Group A & Group B & $p$ value \\
\hline Gastrojejunostomy complications in primary procedures & & \\
Leak (total) (\%) & $10(1.7 \%)$ & $1(0.2 \%)$ & 0.02 \\
Leak requiring reoperation (\%) & $3(0.5 \%)$ & $0(0 \%)$ & 0.26 \\
Anastomotic stricture (\%) & $33(5.7 \%)$ & $3(0.7 \%)$ & $<0.00001$ \\
Marginal ulceration (\%) & $6(1.0 \%)$ & $1(0.2 \%)$ & 0.24 \\
Gastrojejunostomy complications in reoperative procedures & & 0.25 \\
Leak (total) (\%) & $3(4.9 \%)$ & $0(0 \%)$ & 0.5 \\
Leak requiring reoperation (\%) & $2(3.3 \%)$ & $0(0 \%)$ & 0.22 \\
Anastomotic stricture (\%) & $5(8.2 \%)$ & $1(0.2 \%)$ & 0.5 \\
Marginal ulceration (\%) & $2(3.3 \%)$ & $0(0 \%)$ & \\
\hline
\end{tabular}

ulcers can occur early but develop mostly in the long-term, and strictures typically develop during the first trimester after surgery but not during the immediate postoperative period.

In the literature, the incidence of leaks at the GJS after RYGBP varies between extremes of $0 \%$ and more than $8 \%$, but usually between $1 \%$ and $3 \%$ and is reported to depend, among others, on the experience of the surgeon, the surgical approach, the technique of anastomosis (hand sewn versus circular versus linear stapled) and the fact that RYGBP is performed as a primary or re-do procedure [1-11]. Several authors have suggested technical adjuncts to reduce the incidence of leaks, such as reinforcement of the staple line [27], or use of fibrin sealant [28]. Leaks can be totally asymptomatic but can also lead to abscess or severe peritonitis and sepsis and represent a leading cause of death after RYGBP. Symptomatic leaks can require a variety of therapeutic measures, from simple fasting with parenteral nutrition and antibiotics, to stenting or reoperation(s), or a combination of these, leading in any case in a considerable increase in costs.

Anastomotic stricture is another common complication at the GJS, which arises in up to $30 \%$ of cases, although its rate varies widely in the literature [3, 12-23]. The incidence of this complications is also considered by some authors to be related to the operative technique, like linear versus circular stapling [21], absorbable versus non-absorbable sutures [15], and especially to the size of the stapler (21 vs $25 \mathrm{~mm}$ ) when a circular stapler is used [14, 16, 17, 20, 22, 23 ], but stenosis can also develop in a significant number of patients with hand-sewn anastomosis [19]. For instance, Nguyen et al. have noticed that using a 25-mm circular stapler rather than a $21-\mathrm{mm}$ device reduced the stricture rate from $26.8 \%$ to $8.8 \%$ [14], and Suggs et al. found a reduction from 9.4 to $2.9 \%$ [23].

Our overall results compare well with those from the literature for all three complications, with a $1.2 \%$ leak rate and a $3.7 \%$ stricture rate. For strictures, however, comparison is more difficult because the definition of a stricture is different between series. We consider stricture as a complication only if it requires treatment, a restrictive, but clinical, definition, whereas others define it by the diameter of the anastomosis as measured endoscopically or with a barium swallow, with cut-off values up to $10 \mathrm{~mm}$.

In the present study, we were able to reduce the leak and stricture rates by an approximately ten-fold factor after introducing a slight modification of our surgical technique. Our current leak rate $(0.2 \%)$ lies in the lower range of what is currently reported in the literature, and our stricture rate $(0.8 \%)$ is below the usual reported incidence, even by authors suggesting the use of a 25-mm stapler [20, 22, 23]. Both are very likely related to the fact that our more recent anastomotic technique encompasses the horizontal staple line used to divide the stomach and construct the gastric pouch in the circular staple line, thereby avoiding a band of ischemic tissue between the two staple lines. Ischemia and tension on the anastomosis are the two main recognized factors related to anastomotic complications in all surgical settings, and avoiding ischemia certainly is of paramount importance. Other factors, in this series, are unlikely to play a role in this improvement. The two groups were very comparable regarding patient characteristics, except for a slight difference in age in favour of group A. Even if patients who underwent a reoperative RYGBP were excluded, the difference would be the same: $0.2 \%$ versus $1.7 \%(p=0.03)$ for leaks and $0.7 \%$ versus $5.7 \%(p<$ $0.00001)$ for strictures. All anastomosis were performed with a 21-mm circular stapler, which shows that size of the stapler per se is not of utmost importance in the development of anastomotic complications. Tension is not an issue with our technique since we used a retrocolic and retrogastric route for the Roux limb in all but 26 patients in group B (antecolic route), enabling construction of a tension-free anastomosis. The low incidence of marginal ulcers in our series can probably be explained by two factors: the lack of ischemia in the early postoperative period and construction of a very small gastric pouch resulting in very low acidity in the pouch in the long term. 
We could not find a difference in the development of marginal ulceration between our two groups maybe because of its very low overall incidence.

In conclusion, we have shown that good surgical technique significantly decreases the rate of complications at the GJS after laparoscopic Roux-en-Y gastric bypass and that routine creation of a narrow $21 \mathrm{~mm}$ circular stapled anastomosis across the staple line used to create the gastric pouch can be associated with a leak rate of $0.2 \%$ and a stricture rate of $0.8 \%$. These results do not justify recommendations to use a $25-\mathrm{mm}$ circular stapler or other expensive devices like staple line reinforcement or robotics to perform the GJS.

\section{References}

1. Podnos YD, Jimenez JC, Wilson SE, et al. Complications after laparoscopic gastric bypass. Arch Surg. 2003;138:957-61.

2. Dillemans B, Sakran N, Van Cauwenberge S, et al. Standardization of the fully stapled laparoscopic Roux-en-Y gastric bypass for obesity reduces early immediate postoperative morbidity and mortality: a single center study on 2906 patients. Obes Surg. 2009;10:1355-64.

3. Ruiz-de-Adana JC, Lopez-Herrero J, Hernandez-Matias A, et al. Laparoscopic hand-sewn gastrojejunal anastomoses. Obes Surg. 2008;18:1074-6.

4. Agaba EA, Shamseddeen H, Gentles CV. Laparoscopic vs open gastric bypass in the management of morbid obesity: a 7-year retrospective study of 1364 patients from a single center. Obes Surg. 2008;11:1359-63.

5. Ballesta C, Berindoague R, Cabrera M, et al. Management of anastomotic leaks after laparoscopic Roux-en-Y gastric bypass. Obes Surg. 2008;18:623-30.

6. Fobi MAL, Lee H, Holness R, et al. Gastric bypass operation for morbid obesity. World J Surg. 1998;22:925-35.

7. Balsiger BM, Kennedy FP, Abu-Lebdeh HS, et al. Prospective evaluation of Roux-en-Y gastric bypass as primary procedure for medically complicated obesity. Mayo Clin Proc. 2000;75:673-80.

8. Schauer PR, Ikramuddin S, Gourash W, et al. Outcomes after laparoscopic Roux-en-Y gastric bypass for morbid obesity. Ann Surg. 2000;232:515-29.

9. DeMaria EJ, Sugerman HJ, Kellum JM, et al. Results of 281 consecutive total laparoscopic Roux-en-Y gastric bypasses to treat morbid obesity. Ann Surg. 2002;235:640-7.

10. Fernandez AZ, DeMaria EJ, Tichansky DS, et al. Experience with over 3000 open and laparoscopic bariatric procedures. Surg Endosc. 2004;18:193-7.

11. Wittgrove AC, Clark W. Laparoscopic gastric bypass, Roux-en-Y500 patients: technique and results, with 3-60 months follow-up. Obes Surg. 2000;10:233-9.

12. Dolce CJ, Dunnican WJ, Kushnir L, et al. Gastrojejunal strictures after Roux-en-Y gastric bypass with a $21-\mathrm{mm}$ circular stapler. JSLS. 2009;13:306-11.
13. Frutos MD, Lujan J, Garcia A, et al. Gastrojejunal anastomotic stenosis in laparoscopic gastric bypass with a circular stapler $(21 \mathrm{~mm})$ : incidence, treatment and long-term follow-up. Obes Surg 2009;19(12):1631-5.

14. Nguyen NT, Stevens CM, Wolfe BM. Incidence and outcome of anastomotic stricture after laparoscopic gastric bypass. J Gastrointest Surg. 2003;7:997-1003.

15. Vasquez JC, Overby DW, Farrell TM. Fewer gastrojejunostomy strictures and marginal ulcers with absorbable sutures. Surg Endosc. 2009;23:2011-5.

16. Fisher BL, Atkinson JD, Cottam D. Incidence of gastroenterostomy stenosis in laparoscopic Roux-en-Y gastric bypass using 22- or 25-mm circular stapler: a randomised prospective blinded study. Surg Obes Relat Dis. 2007;3:176-9.

17. Gould JC, Garren M, Boll V, et al. The impact of circular stapler diameter on the incidence of gastrojejunostomy stenosis and weight loss following laparoscopic Roux-en-Y gastric bypass. Surg Endosc. 2006;20:1017-20.

18. Schwartz ML, Derw RL, Roiger RW, et al. Stenosis of the gastroenterostomy after laparoscopic gastric bypass. Obes Surg. 2004;14:484-91.

19. Higa KD, Boone KB, Ho T. Complications of the laparoscopic Roux-en-Y gastric bypass: 1040 patients - what have we learned? Obes Surg. 2000;10:509-13.

20. Cottam DR, Fisher B, Sridnar V, et al. The effect of stoma size on weight loss after laparoscopic gastric bypass surgery: results of a blinded randomised controlled trial. Obes Surg. 2009;19:13-7.

21. Leyba JL, Llopis SN, Isaac J, et al. Laparoscopic gastric bypass for morbid obesity - a randomized controlled trial comparing two gastrojejunal anastomosis techniques. JSLS. 2008;12:385-8.

22. Takata MC, Ciovica R, Cello JP, et al. Predictors, treatment, and outcome of gastrojejunostomy stricture after gastric bypass for morbid obesity. Obes Surg. 2007;17:878-84.

23. Suggs WJ, Kouli W, Lupovici M, et al. Complications at gastrojejunostomy after laparoscopic Roux-en-Y gastric bypass: comparison between 21- and 25-mm circular staplers. Surg Obes Relat Dis. 2007;3:508-14.

24. Giusti V, De Lucia A, Di Vetta V, et al. Impact of preoperative teaching on surgical option of patients qualifying for bariatric surgery. Obes Surg. 2004;14:1241-6.

25. Consensus development conference panel. Gastrointestinal surgery for severe obesity: consensus development conference statement. Ann Intern Med. 1991;115:956-61.

26. Consensus sur le traitement de l'obésité en Suisse. Schweiz Med Wochenschr. 1999;129(114):4S-20S.

27. Ibele A, Garren M, Gould J. Effect of circular buttressing material on gastrojejunostomy failure in laparoscopic Roux-en-Y gastric bypass. Surg Obes Relat Dis. 2010;6(1):64-7.

28. Silecchia G, Boru CE, Mouiel J, et al. The use of fibrin sealant to prevent major complications following laparoscopic gastric bypass: results of a multicenter, randomised trial. Surg Endosc. 2008;22:2492-7.

The authors declare no conflict of interest in relation with this manuscript. 